\title{
COVID-19 vaccination in patients with cancer, a rapid review
}

\author{
Laudy Chehade ${ }^{1}$, Jad Zeitoun ${ }^{1}$, Dorothy Lombe ${ }^{2}$, Sheeba Irshad ${ }^{3}$, Mieke Van Hemelrijck ${ }^{4}$, Karen Canfell5,6,7, Richard Sullivan ${ }^{8}$ and \\ Deborah Mukherji1 on behalf of the Cancer and COVID-19 Global Taskforce
}

\author{
${ }^{1}$ Naef K Basile Cancer Institute, American University of Beirut Medical Center, PO Box 11-0236, Riad El Solh, Beirut 1107 2020, Lebanon \\ ${ }^{2}$ Department of Oncology, Cancer Diseases Hospital, Lusaka 10101, Zambia \\ ${ }^{3}$ Comprehensive Cancer Centre, School of Cancer \& Pharmaceutical Sciences, King's College London, London SE1 9RT, UK \\ ${ }^{4}$ Translational Oncology and Urology Research, King's College London, London SE1 9RT, UK \\ ${ }^{5}$ Cancer Research Division, Cancer Council NSW, Sydney, NSW 2011, Australia \\ ${ }^{6}$ School of Public Health, University of Sydney, Sydney, NSW 2006, Australia \\ ${ }^{7}$ Prince of Wales Clinical School, University of New South Wales, Kensington 2052, Australia \\ ${ }^{8}$ Institute of Cancer Policy, King's College London, London, WC2R 2LS, UK
}

\begin{abstract}
Coronavirus disease 2019 (COVID-19) vaccine development and administration have become global priorities since the beginning of the pandemic, particularly for special populations at higher risk of complications and mortality, such as patients with haematologic and solid organ malignancies. This review aims to summarise the current data for COVID19 vaccine efficacy in patients with cancer, suggest priority areas for future research and look at potential disparities at a global level. Although patients diagnosed with or receiving therapy for cancer were excluded from the initial vaccine trials, emerging evidence now supports vaccine safety with potentially diminished immune response in this group. Several studies that evaluated antibody response to COVID-19 vaccination found that patients with solid malignancies had lower serologic response rates compared to healthy controls, but better than patients with haematologic malignancies, who had the lowest seroconversion rates and antibody titres. As anticipated, poor serologic responses have been particularly observed among patients receiving B-cell depleting therapies. The data on cellular response are scarce and conflicting since not all studies have showed a difference between patients with malignancies and healthy subjects. Several questions concerning vaccination remain unanswered and require further exploration, such as response duration, need for response monitoring and rates of breakthrough infections.
\end{abstract}

Keywords: COVID-19 vaccine, haematologic malignancies, solid organ malignancies

\section{Introduction}

Since the coronavirus disease caused by the novel severe acute respiratory syndrome coronavirus 2 (SARS-CoV-2) was declared a pandemic by the World Health Organization on 11 March 2020, vaccine development and administration became a global priority. Certain patient populations are more susceptible to developing severe symptoms with a
Correspondence to: Deborah Mukherji Email: dm25@aub.edu.lb

ecancer 2022, 16:1355

https://doi.org/10.3332/ecancer.2022.1355

Published: $16 / 02 / 2022$

Received: 13/09/2021

Publication costs for this article were supported by ecancer (UK Charity number 1176307).

Copyright: (c) the authors; licensee ecancermedicalscience. This is an Open Access article distributed under the terms of the Creative Commons Attribution License (http:// creativecommons.org/licenses/by/4.0), which permits unrestricted use, distribution, and reproduction in any medium, provided the original work is properly cited. 
worse prognosis following infection, such as those with an advanced age, cardiovascular disease, diabetes, kidney disease, chronic respiratory disease and others [1-6]. Patients diagnosed with a haematologic or solid organ malignancy are also among the populations who are at greatest risk of death or severe complications of coronavirus disease 2019 (COVID-19) [7-10]. In one study, the case fatality rate was $37 \%$ among patients with haematological malignancies and $25 \%$ among patients with solid tumours [11]. In a systematic review and pooled meta-analysis of 52 studies, mortality rate from COVID-19 among patients with cancer was found to be 25.6\% [12]. The increased COVID19 complications encountered by patients with cancer may be due to the immunocompromised state resulting from their disease or antineoplastic therapy. Vaccination programs initially prioritised patients with advanced age or medical co-morbidities associated with severe infection. However, since patients with cancer were excluded from initial vaccine clinical trials, clear guidance on vaccination in patients with malignancy particularly those on active treatment was relatively slow to be produced due to lack of data. Furthermore, in many countries, especially in low-middle income groups, COVID-19 vaccines were simply not available. The aim of this review is to provide an overview of the current data for COVID19-vaccine efficacy and safety in patients with cancer, suggest priority areas for future research and look at potential disparities at a global level.

\section{Vaccine development}

The Spike S Protein is one of the four structural proteins of the virus, and its major role in viral infectivity makes it a target to most vaccines. It is composed of two subunits; the S1 subunit has the receptor-binding domain and mediates the attachment to the ACE2 receptor on the host cells, and the S2 subunit is responsible for viral fusion with cells [13, 14].

The rapid genomic sequencing of the virus, which became available on 11 January 2020, set the path for fast vaccine development [15].

To the date of this review (27 August 2021), ongoing clinical trials are testing 99 vaccines in humans, with more than 75 preclinical trials experimenting on animals [16]. Some of the vaccines were developed using classical technics and others using more novel technology. Unlike conventional vaccines that are based on actual viral particles, new-generation vaccines contain the protein coding sequence responsible for the synthesis of viral antigenic proteins, which stimulate an immune response [13, 14].

Among classical vaccine platforms, inactivated coronavirus vaccines that have reached phase 3 trials include the BBIBP-CorV, CoronaVac and Covaxin [16]. Several new-generation vaccines that have reached phase 3 trials are being administered world-wide, including messenger RNA vaccines and viral vector-based vaccines. The two leading mRNA vaccines are the mRNA-1273, which is authorised for emergency use in multiple countries, and the BTN162b2 vaccine which was granted full Food and Drug Administration (FDA) approval on 23 August 2021. The mRNA vaccines deliver the mRNA sequence encoding viral spike protein, surrounded by lipid nanoparticles to prevent mRNA degradation. The host cells transcribe the mRNA sequence into the viral protein which is expressed by these cells and presented to the immune cells to produce an immune response [17]. As for viral vector-based vaccines, which deliver the vaccine antigens or genes to the receiver's cells, ChAdOx1 nCov-19/AZD1222, Gam-COVID-Vac and Ad26.COV2.S have been developed, all of which have received emergency use authorisation in multiple countries $[14,16]$.

\section{Vaccination in patients with cancer}

Even though COVID-19 vaccines were shown to be effective in preventing hospitalisation and death in registration trials, these trials excluded patients with malignancies who are receiving systemic cytotoxic treatments [18]. Nevertheless, the United States Centers for Disease Control and Prevention (US CDC), National Comprehensive Cancer Network (NCCN), and other professional societies have strongly recommended priority vaccination for immunocompromised individuals including those on active chemotherapy, as these vaccines are not expected to cause major adverse effects and are likely to have a protective effect [19]. Emerging studies have demonstrated good tolerability of the vaccine among patients with cancer, with injection site pain being the most commonly reported side effect [20, 21]. 


\section{Literature search}

We searched PubMed and MEDLINE on 27 August 2021 to identify clinical trials and observational studies to include in this rapid review (see Appendix A). We used the following keywords: 'Antibodies, Viral', 'Antibodies, Neutralizing', 'COVID-19', 'SARS Virus', 'Immunogenicity, Vaccine' and 'Neoplasms'. Additionally, a focused Internet search of the grey literature was done to capture latest updates on guidelines and pre-print articles on vaccine response in patients with cancer. No language restriction was applied to the search. Two authors independently reviewed the list of articles generated by the search and manually selected those that are relevant to the topic and extracted the data. We included studies describing antibody or T-cell response to COVID-19 vaccination in patients with any type of malignancy.

\section{Findings}

\section{Post-vaccination seroconversion rates of patients with solid organ and haematologic malignancies}

The extent to which COVID-19 vaccines are effective in the cancer population is currently being studied. Interim results from the SarsCoV-2 for Cancer Patients (SOAP-02) study which compared the efficacy of the COVID-19 vaccine BNT162b2 among solid cancer patients, haematological cancer patients, and healthy controls showed that only $38 \%$ of patients with solid tumours and $<20 \%$ of patients with haematological malignancies developed positive anti-S IgG titres 21 days after the first dose, whereas $94 \%$ of healthy controls had a positive IgG test [20]. Monin et al [20] also studied the effect of administering a second dose and found that in solid cancer patients, $95 \%$ of those who received a boost at 3 weeks following the initial vaccination dose developed positive anti-S IgG antibodies 2 weeks later, whereas only $30 \%$ of those who did not receive a boost were seropositive. Similar results were found in another study in which only $29 \%$ of solid cancer patients who were receiving systemic chemotherapy, immunotherapy, biological agents or a combination of those, were seropositive after the first dose compared to $84 \%$ of healthy controls; however, after receiving a second dose, $86 \%$ of the patients were seropositive around 2 weeks later [22].

Several studies have looked at the COVID-19 vaccine immune response separately in patient populations with a solid or a haematologic cancer. Patients with a solid cancer had a $94 \%$ seroconversion rate (compared to 100\% in healthy controls) after two doses of COVID-19 vaccine, and anti-spike antibody titres were significantly low among those on chemotherapy [23]. Similar results were found in two other studies where 90\% [24] and 95\% [25] of the solid cancer patients developed positive antibody response post second vaccine dose compared to $100 \%$ of the heathy controls.

With respect to patients diagnosed with a haematologic malignancy, their seroconversion rates are lower than those of healthy controls. One study demonstrated that while seroconversion rate was $100 \%$ in healthy controls 2 weeks post second vaccine dose, it was only $78.6 \%$ patients with multiple myeloma and $88 \%$ in patients with chronic myeloid leukaemia (CML) and myeloproliferative neoplasms (MPNs) [21]. Other studies found that after two doses of vaccine, $39.5 \%$ of patients with chronic lymphocytic leukaemia (CLL) seroconverted versus $100 \%$ of healthy controls [26], and $84.2 \%$ of patients with multiple myeloma had a positive antibody response versus $100 \%$ of the controls [27]. Haematologic malignancy patients were also found to have lower antibody concentrations than healthy controls after two doses of vaccine $[28,29]$.

Some studies have compared vaccine immune response between patients with a haematologic malignancy and those with a solid organ cancer. Their results have shown that patients with a haematologic cancer have lower rates of seroconversion $[20,30]$ and lower median antibody titres compared to those with a solid organ cancer [31, 32]. In one study, compared to patients with a solid tumour, patients with a haematological malignancy had lower rates of seroconversion (77\% versus $98 \%$ ) and lower antibody titres (median, interquartile range (IQR): 832 (24-2,500) versus >2,500 (514-2,500)) after two vaccine doses; both these findings were statistically significant [31]. A study by Thakkar et al [32] also demonstrated that seroconversion rates were significantly lower in patients with a haematologic malignancy compared to a solid tumour (85\% versus $98 \%$ ), especially in patients on anti-CD20 treatment and stem cell transplantation.

It is worth noting that most of the studies relied on anti-spike antibody measurements as a surrogate marker for immunity against COVID19, even though the anti-S IgG level that correlates with a clinically relevant viral inhibition is not yet established. The study by Malard et al 
[33], found that an anti-S IgG level above 3,100 UA/mL correlated with $>30 \%$ neutralising antibodies (NAbs), which is the positive cutoff for NAbs. Moreover, only $46.7 \%$ of their cohort tested above this level after two vaccine doses (compared to $87 \%$ of the controls). Terpos et al [29] considered the neutralising antibody titre cutoff for a positive test to be more than $30 \%$, but a value above $50 \%$ to correspond to a clinically relevant viral inhibition. More studies are needed to define clinically significant neutralising antibody and anti-S IgG titre thresholds.

Data on seroconversion rates and antibody titres across different studies are summarised in Tables 1 and 2 . It is important to note that the studies followed the standard vaccine schedule that is recommended by the manufacturer, and additional data is needed on the immune response following delayed scheduling.

\section{Post-vaccination T-cell response in patients with solid organ and haematologic malignancies}

Cellular immunity also plays a role in the immune response against COVID-19 [34], and the literature shows discrepant results among studies. T-cell response was assessed across different studies using enzyme-linked immunospot (ELISpot) assay or a variation of it. In brief, cytokines released by the stimulated peripheral blood mononuclear cells (PBMCs) are captured by antibodies attached to a membrane and then detected by using a detection antibody. These tests were used to detect interferon gamma (IFN- $\gamma$ ) and interleukin 2 (IL-2) secreting reactive T-cells in response to COVID-19 vaccination.

Monin et al [20] found that following 1 vaccine dose, $82 \%$ of controls, $71 \%$ of patients with solid tumours and $50 \%$ of patients with a haematologic malignancy showed T-cell response. The T-cell response rate in other studies following two vaccine doses was 46\% [30] in patients with a solid cancer and 45\% [30] to 53\% [33] among patients with a haematologic cancer. These results contrast with the findings of the OCTAVE trial, which showed comparable T-cell response rates between healthy controls, patients with solid and haematologic malignancies and haematopoietic stem cell transplant (HSCT) recipients [35].

A positive T-cell response was observed in a number of serologic non-responder patients across several studies [20, 30, 33]. For example, in one study, 17 out of 36 patients who had a T-cell response post-vaccine were seronegative [33], and in another study $26 \%$ of seronegative haematologic patients had a T-cell response [30]. Aleman et al [36] compared B and T-cell response in healthy volunteers, seropositive and seronegative patients with multiple myeloma. Seronegative patients had lower detection rates of spike protein reactive B-cells and CD4 positive T-cell response. All of these findings indicate that despite the fact that some seronegative patients may develop a T-cell response, a significant portion of these patients also appear to lack cellular immunity against COVID-19.

Table 3 summarises the rates of T-cell response to COVID-19 vaccination in patients with cancer across different studies.

\section{Effect of treatment types on vaccine response in patients with cancer}

The impact of antineoplastic treatment on the COVID-19 vaccine immune response was explored across multiple studies, and some therapies were associated with diminishing the vaccine effect, while others were found not to interfere with the response.

Cytotoxic chemotherapy is among the treatment modalities associated with low rates of seroconversion [23, 30-32]. Similarly, anti-CD20 therapies were associated with low seroconversion rates in several studies [26, 28, 30-33], owing to their B-cell depleting effect and impairment of T-cell dependent and independent response, which can last for up to 12 months [37]. In one study, none of the 22 patients who have received anti-CD20 therapy within 12 months of vaccination mounted an antibody response, in contrast with patients who have completed this treatment more than 12 months before vaccination, out of whom 45.5\% developed antibodies [26]. In fact, several authors have advised revaccination for patients on anti-CD20 treatment 6 months after completing their course, or to postpone vaccination till 6 months after the end of treatment [31, 37, 38].

Anti-CD38 treatment, which is used in multiple myeloma, was found to have a negative effect on the immune response. Pimpinelli et al [21] showed that patients on proteasome inhibitors or immunomodulatory drugs (imids)-based treatments without Daratumumab had a $92.9 \%$ seroconversion rate 2 weeks after the second vaccine dose, compared to 50\% in patients on Daratumumab ( $p=0.003)$. Similar results were found by Van Oekelen et al [27] where anti-CD38 treatment was significantly associated with the risk of having a negative antibody response to the vaccine $(p=0.005$, odds ratio $=4.258)$. However, one study did not find a significant association between anti-CD38 therapy and antibody response [30]. 
Table 1. Seroconversion rates in patients with cancer following two doses of COVID-19 vaccine.

\begin{tabular}{|c|c|c|c|c|c|c|c|c|}
\hline \multirow[b]{2}{*}{ Study } & \multirow[b]{2}{*}{ Location } & \multirow[b]{2}{*}{ Vaccine type } & \multirow[b]{2}{*}{ Test used } & \multirow[b]{2}{*}{ Malignancy type } & \multicolumn{4}{|c|}{ Seroconversion rate after two vaccine doses } \\
\hline & & & & & $\begin{array}{l}\text { Solid cancer \% } \\
\qquad(n / N)\end{array}$ & $\begin{array}{l}\text { Haematologic } \\
\text { Malignancy \% } \\
\qquad(n / N)\end{array}$ & $\begin{array}{l}\text { Healthy } \\
\text { control \% } \\
(n / N)\end{array}$ & $p$ value \\
\hline $\begin{array}{l}\text { Massarweh } \\
\text { et al [24] }\end{array}$ & Israel & BNT162b2 & Anti-S IgG & Solid cancer ${ }^{a}$ & $90 \%(92 / 102)$ & & $100 \%(78 / 78)$ & - \\
\hline Addeo et al [31] & $\begin{array}{l}\text { USA and } \\
\text { Europe }\end{array}$ & $\begin{array}{l}\text { mRNA-1273 } \\
\text { or BNT162b2 }\end{array}$ & Anti-S IgG & $\begin{array}{l}\text { Solid and } \\
\text { haematologic } \\
\text { malignancies }^{\text {b }}\end{array}$ & $98 \%(99 / 101)$ & $77 \%(17 / 22)$ & - & 0.002 \\
\hline Monin et al [20] & UK & BNT162b2 & Anti-S IgG & $\begin{array}{l}\text { Solid and } \\
\text { haematologic } \\
\text { malignancies }^{c}\end{array}$ & $95 \%(18 / 19)$ & $60 \%(3 / 5)$ & $100 \%(12 / 12)$ & - \\
\hline $\begin{array}{l}\text { Goshen-Lago } \\
\text { et al [22] }\end{array}$ & Israel & BNT162b2 & Anti-S IgG & Solid cancer ${ }^{d}$ & $86 \%(86 / 100)$ & - & - & - \\
\hline Palich et al [23] & France & BNT162b2 & Anti-S IgG & Solid cancer & $94 \%(210 / 223)$ & - & $100 \%(49 / 49)$ & - \\
\hline Thakkar et al [32] & USA & $\begin{array}{l}\text { mRNA-1273, } \\
\text { BNT162b2 } \\
\text { or AD26. } \\
\text { coV2.S }\end{array}$ & Anti-S IgG & $\begin{array}{l}\text { Solid and } \\
\text { haematologic } \\
\text { malignancies }^{f}\end{array}$ & $98 \%(131 / 134)$ & $85 \%(56 / 66)$ & - & 0.001 \\
\hline Ehmsen et al [30] & Denmark & $\begin{array}{l}\text { mRNA-1273 } \\
\text { or BNT162b2 }\end{array}$ & Anti-S IgG & $\begin{array}{l}\text { Solid and } \\
\text { haematologic } \\
\text { malignancies }^{\mathrm{g}} \\
\end{array}$ & $93 \%(187 / 201)$ & $66 \%(215 / 323)$ & - & 0.004 \\
\hline Malard et al [33] & France & BNT162b2 & Anti-S IgG & $\begin{array}{l}\text { Haematologic } \\
\text { malignancies }^{\text {h }}\end{array}$ & - & 46.7\% (195) & $87 \%(30)$ & 0.0002 \\
\hline $\begin{array}{l}\text { Van Oekelen et al } \\
{[27]}\end{array}$ & USA & $\begin{array}{l}\text { mRNA-1273 } \\
\text { or BNT162b2 }\end{array}$ & Anti-S IgG & Multiple myeloma & - & $84.2 \%(219 / 260)$ & $100 \%(67 / 67)$ & - \\
\hline Lim et al [38] & UK & $\begin{array}{l}\text { ChAdOx1 or } \\
\text { BNT162b2 }\end{array}$ & Anti-S IgG & Lymphomai & - & - & - & - \\
\hline Pimpinelli et al [21] & Italy & BNT162b2 & Anti-S IgG & $\begin{array}{l}\text { Multiple myeloma and } \\
\text { myeloproliferative } \\
\text { malignancies }\end{array}$ & - & $\begin{array}{l}\text { MM: } \\
78.6 \%(33 / 42) \\
\text { MPM: } \\
88 \%(44 / 50)\end{array}$ & $100 \%(36 / 36)$ & $\begin{array}{l}\text { MM: } \\
0.003 \\
\text { MPM: } \\
0.038\end{array}$ \\
\hline Herishanu et al [26] & Israel & BNT162b2 & Anti-S IgG & CLL & - & $52 \%(27 / 52)$ & $100 \%(52 / 52)$ & $<0.001$ \\
\hline Barrière et al [25] & France & BNT162b2 & Anti-S IgG & Solid tumours & $95.2 \%(40 / 42)$ & - & $100 \%(24 / 24)$ & - \\
\hline Kearns et al [35] & UK & $\begin{array}{l}\text { BNT162b2 or } \\
\text { ChAdOx1 }\end{array}$ & Anti-S IgG & $\begin{array}{l}\text { Immunocompromised } \\
\text { patients }^{j}\end{array}$ & $100 \%(47 / 47)$ & $\begin{array}{l}88.9 \%(16 / 18) \\
\text { in patients with } \\
\text { haematologic } \\
\text { malignancies and } \\
88.1 \%(37 / 42) \text { in } \\
\text { HSCT patients }\end{array}$ & $100 \%(93 / 93)$ & - \\
\hline Roeker et al [63] & USA & $\begin{array}{l}\text { BNT162b2 or } \\
\text { mRNA-1273 }\end{array}$ & Anti-S IgG & CLL & - & $52 \%(23 / 44)$ & - & - \\
\hline
\end{tabular}


Table 1. Seroconversion rates in patients with cancer following two doses of COVID-19 vaccine. (Continued)

\begin{tabular}{|c|c|c|c|c|c|c|c|c|}
\hline Terpos et al [29] & Greece & $\begin{array}{l}\text { BNT162b2 or } \\
\text { ChAdOx1 }\end{array}$ & $\begin{array}{l}\text { NAbs } \\
\text { against } \\
\text { SARS- } \\
\text { CoV-2 }\end{array}$ & $\begin{array}{l}\text { Multiple myeloma, } \\
\text { smoldering myeloma, } \\
\text { and monoclonal } \\
\text { gammopathy of } \\
\text { undetermined } \\
\text { significance (MGUS) }\end{array}$ & - & $\begin{array}{l}\text { Neutralising } \\
\text { effect } \geq 30 \% \text { : } \\
71 \%(196 / 276) \\
\text { Neutralising } \\
\text { effect } \geq 50 \% \text { : } \\
57.3 \%(158 / 276)\end{array}$ & $\begin{array}{l}\text { Neutralising } \\
\text { effect } \geq \\
30 \%: 90.3 \% \\
(204 / 226) \\
\text { Neutralising } \\
\text { effect } \\
\geq 50 \%: 81 \% \\
(183 / 226)\end{array}$ & $<0.001$ \\
\hline Parry et al [64] & UK & $\begin{array}{l}\text { BNT162b2 } \\
\text { or ChAdOx1 }\end{array}$ & Anti-S IgG & CLL & - & $\begin{array}{l}\text { Serum samples: } \\
75 \%(9 / 12) \\
\text { Dried blood spot } \\
\text { samples: } 71 \% \\
(39 / 55)\end{array}$ & $\begin{array}{l}\text { Serum } \\
\text { samples: } \\
\text { 100\% (59/59) } \\
\text { Dried blood } \\
\text { spot samples: } \\
97 \%(36 / 37)\end{array}$ & - \\
\hline Benjamini et al [65] & Israel & BNT162b2 & Anti-S IgG & CLL & - & $43 \%(160 / 373)$ & - & - \\
\hline
\end{tabular}

a Gastrointestinal, lung, breast, brain, genitourinary cancers and others

${ }^{b}$ Breast, urologic, gynaecologic, skin, thoracic, gastrointestinal, head and neck, brain and connective tissue cancers. Acute lymphoblastic leukaemia (ALL), CLL, chronic myeloid leukaemia (CML), diffuse large B-cell lymphoma (DLBCL), follicular lymphoma, mucosa-associated lymphoid tissue (MALT) lymphoma, T-cell lymphoma/mycosis fungoides, Hodgkin lymphoma (HL), polycythaemia vera, myeloma

'Gynaecological, breast, renal, prostate, testicular, bladder, skin, thoracic, gastrointestinal and head and neck cancers, glioblastoma, CLL/small lymphocytic lymphoma (SLL), plasma cell myeloma, DLBCL, follicular lymphoma, Burkitt's lymphoma, lymphoplasmacytic lymphoma, mantle cell lymphoma, MALT lymphoma, nodular sclerosing HL, post-renal transplant lymphoproliferative disorder, anaplastic large cell lymphoma, angioimmunoblastic T-cell lymphoma, acute myeloid leukaemia (AML), myelodysplastic syndrome (MDS) or MPN, CML, T-cell precursor ALL, myelofibrosis

d Gastrointestinal, breast, genitourinary, gynaecologic, head and neck, lung, melanoma, neurologic and sarcoma

e Breast, digestive, lung, gynaecological, prostate, bladder, pancreas, kidney, upper aero-digestive tract cancers

${ }^{\mathrm{f} B r e a s t,}$ gastrointestinal, genitourinary, gynaecologic, thoracic, head and neck, skin/musculoskeletal, carcinoma of unknown primary, lymphoid, myeloid and plasma cell malignancies

g Breast, gastrointestinal, urological, gynaecologic, thoracic, skin cancers, CLL/SLL, multiple myeloma, DLBCL, follicular lymphoma, mantle cell lymphoma, marginal zone lymphoma, others

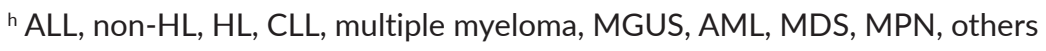

' $\mathrm{HL}$, aggressive B-cell non-HL, indolent B-cell non-HL, natural killer T-cell lymphoma

j Tumour necrosis factor alpha, IL-2 and granulocyte macrophage colony-stimulating factor

k Patients who underwent HSCT were analysed separately from patients with haematologic malignancies

Other therapeutic modalities that were also associated with lower seroconversion rates include HSCT, chimeric antigen receptor (CAR-T) cell treatment, Burton tyrosine kinase inhibitors, Ruxolitinib, Venetoclax, steroids and anti-B-cell maturation antigen bispecific antibody therapy [26-28, 30, 32]. AntiPDL1/PD1, anti-CTLA4, hormonal therapy, anti-Epidermal Growth Factor Receptor 2 and antiangiogenic therapy were not found to affect the rates of seroconversion across studies [23, 28, 30-32].

With regard to T-cell response, steroids and being on current treatment for malignancy were associated with lower T-cell response rates in patients with haematologic malignancies [30, 33]. 
Table 2. Antibody titers in patients with cancer following two doses of COVID-19 vaccine.

\begin{tabular}{|c|c|c|c|c|c|c|c|c|}
\hline \multirow[b]{2}{*}{ Study } & \multirow[b]{2}{*}{ Location } & \multirow[b]{2}{*}{ Vaccine type } & \multirow[b]{2}{*}{ Test type } & \multirow[b]{2}{*}{ Malignancy type } & \multicolumn{4}{|c|}{ Antibody titers, median (IQR) (range) } \\
\hline & & & & & Solid cancer & $\begin{array}{c}\text { Haematologic } \\
\text { malignancy }\end{array}$ & Healthy controls & $p$ value \\
\hline $\begin{array}{l}\text { Massarweh } \\
\text { et al [24] }\end{array}$ & Israel & BTN162b2 & $\begin{array}{l}\text { Anti-S } \\
\lg G\end{array}$ & Solid cancer & $\begin{array}{l}1,931 \\
(509-4,386) \\
(0.3-53,088)\end{array}$ & & $\begin{array}{l}7,160 \\
(3,129-11,241) \\
(442-27,568)\end{array}$ & $<0.001$ \\
\hline Lim et al [38] & UK & $\begin{array}{l}\text { ChAdOx1 or } \\
\text { BTN162b2 }\end{array}$ & $\begin{array}{l}\text { Anti-S } \\
\lg G\end{array}$ & Lymphoma & - & $\begin{array}{l}2.5 \mathrm{BAU} / \\
\mathrm{mL}(95 \% \mathrm{Cl} \\
1.1-5.8) \text { for } \\
\text { participants on } \\
\text { treatment and } \\
141.8 \mathrm{BAU} / \mathrm{mL} \\
(75.6-266.0) \text { for } \\
\text { participants not } \\
\text { on treatment. }\end{array}$ & $\begin{array}{l}2,339 \mathrm{BAU} / \mathrm{mL} \\
(1,923-2,844) \\
\text { in those who } \\
\text { took BNT162b2; } \\
199 \mathrm{BAU} / \mathrm{mL} \\
(140-282) \text { in } \\
\text { those who took } \\
\text { ChAdOx1 }\end{array}$ & - \\
\hline $\begin{array}{l}\text { Addeo } \\
\text { et al [31] }\end{array}$ & $\begin{array}{l}\text { USA and } \\
\text { Europe }\end{array}$ & $\begin{array}{l}\text { mRNA- } \\
1273 \text { or BNT162b2 }\end{array}$ & $\begin{array}{l}\text { Anti-S } \\
\lg G\end{array}$ & $\begin{array}{l}\text { Solid and } \\
\text { haematologic } \\
\text { malignancies }^{\mathrm{a}}\end{array}$ & $\begin{array}{l}>2,500 \\
(514-2,500)\end{array}$ & $832(24-2,500)$ & - & 0.029 \\
\hline $\begin{array}{l}\text { Palich et al } \\
\text { [23] }\end{array}$ & France & BNT162b2 & $\begin{array}{l}\text { Anti-S } \\
\lg G\end{array}$ & Solid cancer ${ }^{b}$ & $\begin{array}{l}252 \text { (Roche } \\
\text { Elecsys assay); } \\
4,443 \text { Abbott } \\
\text { Alinity Assay }\end{array}$ & - & $\begin{array}{l}2,517 \text { (Roche } \\
\text { Elecsys assay); } \\
\text { 13,285 Abbott } \\
\text { Alinity assay }\end{array}$ & $\begin{array}{l}<0.01 \text { for } \\
\text { both } \\
\text { assays }\end{array}$ \\
\hline $\begin{array}{l}\text { Thakkar et al } \\
\text { [32] }\end{array}$ & USA & $\begin{array}{l}\text { mRNA-1273, or } \\
\text { BNT162b2 or } \\
\text { AD26.COV2.S }\end{array}$ & $\begin{array}{l}\text { Anti-S } \\
\lg G\end{array}$ & $\begin{array}{l}\text { Solid and } \\
\text { haematologic } \\
\text { malignancies }^{c}\end{array}$ & 7,858 & 2,528 & - & 0.013 \\
\hline $\begin{array}{l}\text { Van Oekelen } \\
\text { et al [27] }\end{array}$ & USA & $\begin{array}{l}\text { mRNA-1273 or } \\
\text { BNT162b2 }\end{array}$ & $\begin{array}{l}\text { Anti-S } \\
\lg G\end{array}$ & Multiple myeloma & - & $\begin{array}{l}149(5-7,882 \\
A U / m L)\end{array}$ & $\begin{array}{l}300(21-3,335 \\
\mathrm{AU} / \mathrm{mL})\end{array}$ & $<0.0001$ \\
\hline $\begin{array}{l}\text { Pimpinelli } \\
\text { et al [21] }\end{array}$ & Italy & BNT162b2 & $\begin{array}{l}\text { Anti-S } \\
\lg G\end{array}$ & $\begin{array}{l}\text { Multiple } \\
\text { myeloma and } \\
\text { myeloproliferative } \\
\text { malignancies }\end{array}$ & - & $\begin{array}{l}\text { MM: } 106.7 \mathrm{AU} / \\
\mathrm{mL} \\
\text { MPM: } \\
172.9 \mathrm{AU} / \mathrm{mL}\end{array}$ & $353.3 \mathrm{AU} / \mathrm{mL}$ & $\begin{array}{l}\text { MM: } \\
0.003 \\
\text { MPM: } \\
0.049\end{array}$ \\
\hline $\begin{array}{l}\text { Herishanu } \\
\text { et al [26] }\end{array}$ & Israel & BNT162b2 & $\begin{array}{l}\text { Anti-S } \\
\operatorname{lgG}\end{array}$ & CLL & - & $0.824 \mathrm{U} / \mathrm{mL}$ & $1,084 \mathrm{U} / \mathrm{mL}$ & $<0.001$ \\
\hline $\begin{array}{l}\text { Barrière } \\
\text { et al [25] }\end{array}$ & France & BNT162b2 & $\begin{array}{l}\text { Anti-S } \\
\lg G\end{array}$ & Solid tumours & $245.2 \mathrm{UI} / \mathrm{mL}$ & - & $2,517 \mathrm{UI} / \mathrm{mL}$ & $<0.001$ \\
\hline $\begin{array}{l}\text { Maneikis } \\
\text { et al [28] }\end{array}$ & Lithuania & BNT162b2 & $\begin{array}{l}\text { Anti-S } \\
\operatorname{lgG}\end{array}$ & $\begin{array}{l}\text { Haematological } \\
\text { malignancies }\end{array}$ & - & 6,961 AU/mL & $21,395 \mathrm{AU} / \mathrm{mL}$ & $<0.0001$ \\
\hline $\begin{array}{l}\text { Kearns et al } \\
\text { [35] }\end{array}$ & UK & $\begin{array}{l}\text { BNT162b2 or } \\
\text { ChAdOx1 }\end{array}$ & $\begin{array}{l}\text { Anti-S } \\
\lg G\end{array}$ & $\begin{array}{l}\text { Immuno } \\
\text { compromised } \\
\text { patients }^{d}\end{array}$ & $\begin{array}{l}4,101 \\
(655-10,819 \\
U / m L)\end{array}$ & $\begin{array}{l}1,011.5 \\
(17.3-3,877 \\
\mathrm{U} / \mathrm{mL}) \text { in } \\
\text { patients with } \\
\text { haematologic } \\
\text { malignancies and } \\
980(91.9-5,129 \\
\mathrm{U} / \mathrm{mL}) \text { in } \mathrm{HSCT} \\
\text { patients }^{\mathrm{e}}\end{array}$ & $\begin{array}{l}11,514(3,324- \\
23,302 \mathrm{U} / \mathrm{mL})\end{array}$ & - \\
\hline
\end{tabular}


Table 2. Antibody titers in patients with cancer following two doses of COVID-19 vaccine. (Continued)

\begin{tabular}{|l|l|l|l|l|l|l|l|}
\hline $\begin{array}{l}\text { Parry et al } \\
{[64]}\end{array}$ & UK & $\begin{array}{l}\text { BNT162b2 or } \\
\text { ChAdOx1 }\end{array}$ & $\begin{array}{l}\text { Anti-S } \\
\text { IgG }\end{array}$ & CLL & - & $53 \mathrm{U} / \mathrm{mL}$ & $3,900 \mathrm{U} / \mathrm{mL}$ \\
\hline
\end{tabular}

${ }^{a}$ Breast, urologic, gynaecologic, skin, thoracic, gastrointestinal, head and neck, brain and connective tissue cancers. ALL, CLL, CML, DLBCL, follicular lymphoma, MALT lymphoma, T-cell lymphoma/mycosis fungoides, HL, polycythaemia vera, myeloma

${ }^{\mathrm{b}}$ Breast, digestive, lung, gynaecological, prostate, bladder, pancreas, kidney, upper aero-digestive tract cancers

' Breast, gastrointestinal, genitourinary, gynaecologic, thoracic, head and neck, skin/musculoskeletal, carcinoma of unknown primary, lymphoid, myeloid and plasma cell malignancies

dTumour necrosis factor alpha, IL-2 and granulocyte macrophage colony-stimulating factor

e Patients who underwent HSCT were analysed separately from patients with haematologic malignancies

\section{Areas requiring further research and exploration}

\section{Duration of vaccine response}

The duration of the immune response to the COVID-19 vaccine has yet to be explored, as more time passes since the initial vaccine rollout. Ehmsen et al [30] showed a decline in titres and seropositivity rates from $93 \%$ at 36 days post-vaccination to $86 \%$ at 3 months post vaccination for patients with a solid cancer, and from $66 \%$ to $53 \%$ for patients with a haematologic malignancy. Only one patient of the cohort, initially seronegative at 36 days, became positive after 3 months. As different vaccine types exist in the market, some being more effective than others, the question of combining different vaccine types to optimise response remains unanswered.

\section{Heterologous COVID-19 vaccination}

The use of different vaccines types for first and second dose has been investigated in some studies that did not involve patients with cancer. The aim of heterologous vaccination is to avoid shortage of one type of vaccine, which might slow down vaccine rollout, while maintaining efficacy. The Com-COV2 study involved 1,072 participants who received BNT162b2 or ChAdOx1 vaccines for their first dose were randomised to receive a homologous vaccine, mRNA-1273 or NVX-CoV2373 vaccine as their second dose after 8 to 12 weeks. The immunogenicity of mRNA-1273 as second dose following ChAdOx1 or BNT162b2 was not inferior to that of homologous vaccine schedule [39]. In another phase II study, BNT162b2 was administered as second dose to participants primed with ChAdOx1, which was shown to result in a strong humoral and cellular response and mild reactogenicity [40]. Further exploration of this topic is needed, with studies involving patients with cancer.

\section{Third dose recommendation and need for response monitoring}

Although vaccination is necessary for patients with cancer, the optimal timing for vaccine administration during treatment and the need for response monitoring remain to be determined. Some authors have recommended assessing vaccine response and to revaccinate after completion of treatment [33, 38]. As of August 2021, the FDA [41], CDC [42] and NCCN [43] have authorised a third vaccine dose for patients with moderate-to-severe immunodeficiencies, including patients with solid and haematologic malignancies on active treatment, CAR-T cell and HSCT recipients, given that these patients might not be able to mount sufficient immunity following the regular vaccine series. The UK Joint Committee on Vaccination and Immunisation has also advised having a third dose part of the primary vaccination series for patients with severe immunosuppression [44]. Although data proving the efficacy of a third vaccine dose in solid organ transplant recipients [45] is available, such data is limited for patients with cancer and may be the topic of future investigations. In a cohort of 20 patients with cancer, 16 patients had improved neutralising antibody titres following a third BNT162b2 vaccine dose, but there was no improvement in circulating spike-specific T cell frequencies [46]. In a recent study, 88 patients with cancer received a COVID-19 booster vaccine 28 days following the standard series. $56 \%$ of the patients who were initially seronegative seroconverted after the booster shot $(p=0.000062)$ and those with a haematological malignancy had a smaller change in anti-S IgG titres post booster compared to patients with solid organ tumours (10,034 versus 22,686 $\mathrm{AU} / \mathrm{mL}, p=0.00263$ ) [47]. 
Table 3. T-cell response in patients with cancer following 2 doses of COVID-19 vaccine.

\begin{tabular}{|c|c|c|c|c|c|c|c|c|}
\hline \multirow[b]{2}{*}{ Study } & \multirow[b]{2}{*}{ Location } & \multirow[b]{2}{*}{ Vaccine type } & \multirow[b]{2}{*}{ Test type } & \multirow[b]{2}{*}{ Malignancy type } & \multicolumn{4}{|c|}{ T-cell response rate } \\
\hline & & & & & Solid & Haematologic & $\begin{array}{l}\text { Healthy } \\
\text { control }\end{array}$ & $p$ value \\
\hline $\begin{array}{l}\text { Monin et al } \\
\text { [20] }\end{array}$ & UK & BNT162b2 & $\begin{array}{l}\text { Fluorospot assay for } \\
\text { IFN } \gamma \text {-producing and IL- } \\
\text { 2-producing SARS-CoV- } \\
\text { 2-reactive T-cells }\end{array}$ & $\begin{array}{l}\text { Solid and haematologic } \\
\text { malignancies }^{\text {a }}\end{array}$ & $\begin{array}{l}88 \% \\
(14 / 16)\end{array}$ & $75 \%(3 / 4)$ & $\begin{array}{l}100 \% \\
(3 / 3)\end{array}$ & - \\
\hline $\begin{array}{l}\text { Ehmsen et al } \\
\text { [30] }\end{array}$ & Denmark & $\begin{array}{l}\text { mRNA- } \\
1273 \text { and } \\
\text { BNT162b2 }\end{array}$ & $\begin{array}{l}\text { Whole blood IFN- } \gamma \\
\text { release immunoassay }\end{array}$ & $\begin{array}{l}\text { Solid and haematologic } \\
\text { malignancies }^{\text {b }}\end{array}$ & $\begin{array}{l}46 \% \\
(92 / 201)\end{array}$ & $45 \%(144 / 323)$ & - & - \\
\hline $\begin{array}{l}\text { Malard et al } \\
\text { [33] }\end{array}$ & France & BNT162b2 & $\begin{array}{l}\text { IFN- } \gamma \text { production } \\
\text { measurement on } \\
\text { PBMCs (ELISPOT assay) }\end{array}$ & $\begin{array}{l}\text { Haematologic } \\
\text { malignancies }^{c}\end{array}$ & - & $53 \%(36 / 68)$ & - & - \\
\hline $\begin{array}{l}\text { Aleman et al } \\
{[36]}\end{array}$ & USA & $\begin{array}{l}\text { mRNA- } \\
1273 \text { and } \\
\text { BNT162b2 }\end{array}$ & $\begin{array}{l}\text { IFN- } \gamma, \text { TNF- } a, \text { IL-2 and } \\
\text { GM-CSFd measurement } \\
\text { within CD4+ and } \\
\text { CD8+ T-cells through } \\
\text { intracellular cytokine } \\
\text { staining }\end{array}$ & Multiple myeloma & - & $\begin{array}{l}\text { CD4+ response } \\
\text { in } 96 \%(25 / 26) \\
\text { of seropositive } \\
\text { patients and } \\
35 \%(6 / 17) \text { of } \\
\text { seronegative } \\
\text { patients }\end{array}$ & $100 \%$ & $<0.001$ \\
\hline Study & Location & Vaccine type & Test type & Malignancy type & \multicolumn{4}{|c|}{ IFN- $\gamma$ secreting T-cell/10 $10^{6}$ PBMCs, median (IQR) } \\
\hline $\begin{array}{l}\text { Kearns et al } \\
\text { [35] }\end{array}$ & UK & $\begin{array}{l}\text { BNT162b2 } \\
\text { and } \\
\text { ChAdOx1 }\end{array}$ & $\begin{array}{l}\text { Spike specific IFN- } \gamma \text { T } \\
\text { cell response (Oxford } \\
\text { Immunotec T-SPOT } \\
\text { Discovery SARS-CoV-2 } \\
\text { assay) }\end{array}$ & $\begin{array}{l}\text { Immunocompromised } \\
\text { patients }\end{array}$ & $\begin{array}{l}32 \% \\
(8-112)\end{array}$ & $\begin{array}{l}54(20-164) \text { in } \\
\text { patients with } \\
\text { haematologic } \\
\text { malignancies } \\
\text { and } 32 \text { (8-108) } \\
\text { in HSCT } \\
\text { patients }\end{array}$ & $\begin{array}{l}60 \% \\
(20- \\
136)\end{array}$ & \\
\hline
\end{tabular}

${ }^{a}$ Gynaecological, breast, renal, prostate, testicular, bladder, skin, thoracic, gastrointestinal and head and neck cancers, glioblastoma, CLL/ SLL, plasma cell myeloma, DLBCL, follicular lymphoma, Burkitt's lymphoma, lymphoplasmacytic lymphoma, mantle cell lymphoma, MALT lymphoma, nodular sclerosing HL, post-renal transplant lymphoproliferative disorder, anaplastic large cell lymphoma, angioimmunoblastic T-cell lymphoma, AML, MDS or MPN, CML, T-cell precursor ALL, myelofibrosis

${ }^{\mathrm{b}}$ Breast, gastrointestinal, urological, gynaecologic, thoracic, skin cancers, CLL/SLL, multiple myeloma, DLBCL, follicular lymphoma, mantle cell lymphoma, marginal zone lymphoma, others

${ }^{c} \mathrm{ALL}$, non-HL, HL, CLL, multiple myeloma, MGUS, AML, MDS, MPN, others

${ }^{\mathrm{d}}$ Tumour necrosis factor alpha, IL-2 and granulocyte macrophage colony-stimulating factor

e Inflammatory arthritis, antineutrophil cytoplasmic antibodies-associated vasculitis, end stage kidney disease patients requiring with or without immunosuppression, hepatic disease, inflammatory bowel disease, solid cancer, haematologic malignancy, HSCT

\section{Breakthrough infections in patients with cancer and vaccine efficacy against emerging variants}

Data are emerging on the rate of breakthrough infections among vaccinated patients with cancer. A study by Heudel et al [48] found the rate of infection to be $0.4 \%$ in patients who received two doses and $5 \%$ in those who took one dose $(p<0.0001) .12 .5 \%$ of the patients who got COVID-19 infection died, making the overall mortality rate $0.7 \%$ in patients with a haematologic malignancy and $0.08 \%$ in patients with solid 
tumours. In another prospective cohort study, 9 out of 885 fully vaccinated haematologic malignancy patients had COVID-19 infection; 6 required oxygen supplementation and 3 died [28]. In the study by Van Oekelen et al [27] 10 out of 260 patients with multiple myeloma developed COVID-19 infection after receiving at least 1 dose of mRNA vaccine, 3 of which have developed the infection after getting 2 doses. Three of the ten patients required hospitalisation and one patient required intensive care and passed away [27]. It is important to monitor the rate of breakthrough infections, particularly that new variants of COVID-19 are emerging, and the effect of the available vaccines against them is still uncertain.

\section{Viral shedding in vaccinated patients post infection}

Several studies about the duration of viral shedding in patients infected with COVID-19 have been conducted. The main goal of these studies was to determine the approximate number of days during which infected patients must isolate themselves after being diagnosed, in an attempt to decrease the transmission rates. The duration of viral shedding differed based on the severity of the infection. While most infected patients shed infectious particles for a maximum of 8 days $[49,50]$, some hospitalised patients with high viral loads continue to shed viable viral particles for more than 2 weeks [51, 52]. Patients diagnosed with solid or haematological malignancies are usually on immunosuppressive therapies and therefore tend to shed viral particles for longer periods. Aydillo et al [53] showed that cancer patients receiving treatments like HSCT and CAR-T T-cell therapy may shed viable infectious particles for 2 or more months after their initial diagnosis with COVID-19. One immunocompromised patient with CLL continued shedding particles even 70 days post diagnosis [54]. These findings support the CDC guidelines for cancer patients, which state that cancer patients receiving chemotherapy should isolate for a minimum of 20 days after their first positive PCR test [55]. Vaccination against COVID-19 was shown to significantly reduce viral load and shedding in patients with breakthrough infections $[56,57]$. However, the literature still lacks data about the effect of vaccines on the viral shedding in immunocompromised patients with solid or haematological malignancies specifically.

\section{COVID-19 prevention priorities for low-income countries}

Currently, vaccination rates are much higher in high-income countries compared to low- and middle-income countries (LMICs) [58]. The continuous widening of the gap in vaccination rates between high-income countries and other groups may be explained by several factors. A recent regression analysis showed that LMICs had significantly lower vaccination coverage and vaccination policy strength relative to high-income countries 6 months after the start of vaccine distribution [59]. In addition, other factors explaining the lower vaccination rates in LMICs may include vaccine hesitancy among the population, lack of awareness, spread of unreliable information about vaccine safety, and low socioeconomic status $[60,61]$. Similar factors also seem to play a role in lowering vaccination rates in cancer patients in these countries [62]. Taking all this and the increased susceptibility of cancer patients to higher COVID-19 morbidity and mortality into account, the maintenance of strict precautions in this population of patients in LMICs is extremely important.

\section{Conclusion}

COVID-19 mitigation through vaccination is crucial in patients with solid organ and haematologic malignancies, as they are at high risk of morbidity and mortality from this infection. Despite the fact that patients with cancer were excluded from original vaccine trials, recent studies have showed that these vaccines are safe in this broad population. However, seroconversion is variable and depends on the type of malignancy and treatment, as patients with solid malignancies have lower seroconversion rates than healthy controls, but better than patient with haematologic malignancies. Cellular immunity was also observed to follow a similar trend in some studies, and found to be comparable among these three cohorts in one report. Therefore, a third dose (booster) of the vaccine is likely beneficial for cancer patients, although efficacy data in this population is limited. Response monitoring may also be needed, particularly in patients who are severely immunocompromised. In low and many middle-income countries where the rate and coverage of COVID-19 vaccination is slow, cancer patients will continue to be at higher risk of morbidity and mortality. 


\section{Conflicts of interest}

The authors do not report any relevant conflicts of interest.

\section{Funding}

The Cancer and COVID-19 Global Taskforce (https://covidcancertaskforce.org) is funded through UK Research and Innovation as part of the Global Challenges Research Fund; Research for Health in Conflict in the Middle East and North Africa (R4HC-MENA) project, grant number ES/P010962/1.

\section{Reference}

1. Huang S, Wang J, and Liu F, et al (2020) COVID-19 patients with hypertension have more severe disease: a multicenter retrospective observational study Hypertens Res43(8): 824-831 https://doi.org/10.1038/s41440-020-0485-2 PMID: 32483311 PMCID: 7261650

2. Guo W, Li M, and Dong Y, et al (2020) Diabetes is a risk factor for the progression and prognosis of COVID-19 Diabetes Metab Res Rev 36 e3319 https://doi.org/10.1002/dmrr.3319

3. Zhou F, Yu T, and Du R, et al (2020) Clinical course and risk factors for mortality of adult inpatients with COVID-19 in Wuhan, China: a retrospective cohort study Lancet 395(10229) 1054-1062 https://doi.org/10.1016/S0140-6736(20)30566-3 PMID: 32171076 PMCID: $\underline{7270627}$

4. Ebinger JE, Achamallah N, and Ji H, et al (2020) Pre-existing traits associated with Covid-19 illness severity PLoS One 15(7) e0236240 https://doi.org/10.1371/journal.pone.0236240 PMID: 32702044 PMCID: 7377468

5. van Gerwen M, Alsen M, and Little C, et al (2021) Risk factors and outcomes of COVID-19 in New York City; a retrospective cohort study J Med Virol 93(2) 907-915 https://doi.org/10.1002/jmv.26337

6. Soares RCM, Mattos LR, and LM Raposo (2020) Risk factors for hospitalization and mortality due to COVID-19 in Espírito Santo State, Brazil Am J Trop Med Hyg 103(3) 1184-1190 https://doi.org/10.4269/ajtmh.20-0483 PMID: 32682453 PMCID: 7470570

7. Williamson EJ, Walker AJ, and Bhaskaran K, et al (2020) Factors associated with COVID-19-related death using OpenSAFELY Nature 584(7821) 430-436 https://doi.org/10.1038/s41586-020-2521-4 PMID: 32640463 PMCID: 7611074

8. Liang W, Guan W, and Chen R, et al (2020) Cancer patients in SARS-CoV-2 infection: a nationwide analysis in China Lancet Oncol 21(3) 335-337 https://doi.org/10.1016/S1470-2045(20)30096-6 PMID: 32066541 PMCID: 7159000

9. Tian J, Yuan X, and Xiao J, et al (2020) Clinical characteristics and risk factors associated with COVID-19 disease severity in patients with cancer in Wuhan, China: a multicentre, retrospective, cohort study Lancet Oncol 21(7) 893-903 https://doi.org/10.1016/S14702045(20)30309-0 PMID: 32479790 PMCID: 7259911

10. Kuderer NM, Choueiri TK, and Shah DP, et al (2020) Clinical impact of COVID-19 on patients with cancer (CCC19): a cohort study Lancet 395(10241) 1907-1918 https://doi.org/10.1016/S0140-6736(20)31187-9 PMID: 32473681 PMCID: 7255743

11. Mehta V, Goel S, and Kabarriti R, et al (2020) Case fatality rate of cancer patients with COVID-19 in a New York Hospital System Cancer Discov 10(7) 935-941 https://doi.org/10.1158/2159-8290.CD-20-0516 PMID: 32357994 PMCID: 7334098

12. Saini KS, Tagliamento M, and Lambertini M, et al (2020) Mortality in patients with cancer and coronavirus disease 2019: a systematic review and pooled analysis of 52 studies Eur J Cancer 139 43-50 https://doi.org/10.1016/j.ejca.2020.08.011 PMID: 32971510 PMCID: $\underline{7467090}$ 
13. Han X, Xu P, and Ye Q (2021) Analysis of COVID-19 vaccines: types, thoughts, and application J Clin Lab Anal 35 e23937 https://doi. org/10.1002/jcla.23937 PMID: 34396586 PMCID: 8418485

14. Karpiński TM, Ożarowski M, and Seremak-Mrozikiewicz A, et al (2021) The 2020 race towards SARS-CoV-2 specific vaccines Theranostics 11(4) 1690-1702 https://doi.org/10.7150/thno.53691

15. Wang J, Peng $\mathrm{Y}$, and $\mathrm{Xu} \mathrm{H}$, et al (2020) The COVID-19 vaccine race: challenges and opportunities in vaccine formulation AAPS PharmSciTech 21(6) 225 https://doi.org/10.1208/s12249-020-01744-7 PMID: 32761294 PMCID: 7405756

16. Zimmer C, Corum J, and Wee S, et al (2021) Coronavirus Vaccine Tracker [https://www.nytimes.com/interactive/2020/science/coronavirus-vaccine-tracker.html]

17. Pardi N, Hogan MJ, and Porter FW, et al (2018) mRNA vaccines-a new era in vaccinology Nat Rev Drug Discov 17(4) 261-279 https:// doi.org/10.1038/nrd.2017.243 PMID: 29326426 PMCID: 5906799

18. Friese CR, Choueiri TK, and Duma N, et al (2021) Care without a compass: including patients with cancer in COVID-19 studies Cancer Cell 39(7) 895-896 https://doi.org/10.1016/j.ccell.2021.04.006 PMID: 33894136 PMCID: 8049404

19. Hwang JK, Zhang T, and Wang AZ, et al (2021) COVID-19 vaccines for patients with cancer: benefits likely outweigh risks J Hematol Oncol 14(1) 38 https://doi.org/10.1186/s13045-021-01046-w PMID: 33640005 PMCID: 7910769

20. Monin L, Laing AG, and Muñoz-Ruiz M, et al (2021) Safety and immunogenicity of one versus two doses of the COVID-19 vaccine BNT162b2 for patients with cancer: interim analysis of a prospective observational study Lancet Oncol 22(6) 765-778 https://doi. org/10.1016/S1470-2045(21)00213-8 PMID: 33930323 PMCID: 8078907

21. Pimpinelli F, Marchesi F, and Piaggio G, et al (2021) Fifth-week immunogenicity and safety of anti-SARS-CoV-2 BNT162b2 vaccine in patients with multiple myeloma and myeloproliferative malignancies on active treatment: preliminary data from a single institution $J$ Hematol Oncol 14(1) 81 https://doi.org/10.1186/s13045-021-01090-6 PMID: 34001183 PMCID: 8128283

22. Goshen-Lago T, Waldhorn I, and Holland R, et al (2021) Serologic status and toxic effects of the sars-cov-2 bnt162b2 vaccine in patients undergoing treatment for cancer JAMA Oncology 7 1507-1513 https://doi.org/10.1001/jamaoncol.2021.2675 PMID: 34236381 PMCID: 8267843

23. Palich R, Veyri M, and Vozy A, et al (2021) High seroconversion rate but low antibody titers after two injections of BNT162b2 (Pfizer-BioNTech) vaccine in patients treated by chemotherapy for solid cancers Ann Oncol 32 1294-1295 https://doi.org/10.1016/j. annonc.2021.06.018 PMID: 34171494 PMCID: 8217700

24. Massarweh A, Eliakim-Raz N, and Stemmer A, et al (2021) Evaluation of seropositivity following BNT162b2 messenger RNA vaccination for SARS-CoV-2 in patients undergoing treatment for cancer JAMA Oncol 7 1133-1140 https://doi.org/10.1001/jamaoncol.2021.2155 PMID: 34047765 PMCID: 8164144

25. Barrière J, Chamorey E, and Adjtoutah Z, et al (2021) Impaired immunogenicity of BNT162b2 anti-SARS-CoV-2 vaccine in patients treated for solid tumors Ann Oncol 32(8) 1053-1055 https://doi.org/10.1016/j.annonc.2021.04.019 PMID: 33932508 PMCID: 8080507

26. Herishanu Y, Avivi I, and Aharon A, et al (2021) Efficacy of the BNT162b2 mRNA COVID-19 vaccine in patients with chronic lymphocytic leukemia Blood 137(23) 3165-3173 https://doi.org/10.1182/blood.2021011568 PMID: 33861303 PMCID: 8061088

27. Van Oekelen O, Gleason CR, and Agte S, et al (2021) Highly variable SARS-CoV-2 spike antibody responses to two doses of COVID-19 RNA vaccination in patients with multiple myeloma Cancer Cell 39(8) 1028-1030 https://doi.org/10.1016/j.ccell.2021.06.014 PMID: 34242572 PMCID: 8238657

28. Maneikis K, Šablauskas K, and Ringelevičiūtė U, et al (2021) Immunogenicity of the BNT162b2 COVID-19 mRNA vaccine and early clinical outcomes in patients with haematological malignancies in Lithuania: a national prospective cohort study Lancet Haematol 8(8) e583-e592 https://doi.org/10.1016/S2352-3026(21)00169-1 PMID: 34224668 PMCID: 8253543 
29. Terpos E, Gavriatopoulou M, and Ntanasis-Stathopoulos I, et al (2021) The neutralizing antibody response post COVID-19 vaccination in patients with myeloma is highly dependent on the type of anti-myeloma treatment Blood Cancer J 11(8) 138 https://doi.org/10.1038/ s41408-021-00530-3 PMID: 34341335 PMCID: 8327056

30. Ehmsen S, Asmussen A, and Jeppesen SS, et al (2021) Antibody and T cell immune responses following mRNA COVID-19 vaccination in patients with cancer Cancer Cell 39(8) 1034-1036 https://doi.org/10.1016/j.ccell.2021.07.016 PMID: 34348121 PMCID: 8313483

31. Addeo A, Shah PK, and Bordry N, et al (2021) Immunogenicity of SARS-CoV-2 messenger RNA vaccines in patients with cancer Cancer Cell 39 1091-1098 https://doi.org/10.1016/j.ccell.2021.06.009 PMID: 34214473 PMCID: 8218532

32. Thakkar A, Gonzalez-Lugo JD, and Goradia N, et al (2021) Seroconversion rates following COVID-19 vaccination among patients with cancer Cancer Cell 39(8) 1081-1090.e2 https://doi.org/10.1016/j.ccell.2021.06.002 PMID: 34133951 PMCID: 8179248

33. Malard F, Gaugler B, and Gozlan J, et al (2021) Weak immunogenicity of SARS-CoV-2 vaccine in patients with hematologic malignancies Blood Cancer J 11(8) 142 https://doi.org/10.1038/s41408-021-00534-z PMID: 34376633 PMCID: 8353615

34. DiPiazza AT, Graham BS, and Ruckwardt TJ (2021) T cell immunity to SARS-CoV-2 following natural infection and vaccination Biochem Biophys Res Commun 538 211-217 https://doi.org/10.1016/j.bbrc.2020.10.060

35. Kearns P, Siebert S, and Gaskell C, et al (2021) Examining the Immunological Effects of COVID-19 Vaccination in Patients with Conditions Potentially Leading to Diminished Immune Response Capacity - The OCTAVE Trial

36. Aleman A, Upadhyaya B, and Tuballes K, et al (2021) Variable cellular responses to SARS-CoV-2 in fully vaccinated patients with multiple myeloma Cancer Cell 39(11) 1442-1444 https://doi.org/10.1016/j.ccell.2021.09.015 PMID: 34706273 PMCID: 8523488

37. Houot R, Levy R, and Cartron G, et al (2020) Could anti-CD20 therapy jeopardise the efficacy of a SARS-CoV-2 vaccine? Eur J Cancer 136 4-6 https://doi.org/10.1016/j.ejca.2020.06.017 PMID: 32619884 PMCID: 7315961

38. Lim SH, Campbell N, and Johnson M, et al (2021) Antibody responses after SARS-CoV-2 vaccination in patients with lymphoma Lancet Haematol 8 e542-e544 https://doi.org/10.1016/S2352-3026(21)00199-X PMID: 34224667 PMCID: 8253538

39. Stuart AS, Shaw RH, and Liu X, et al (2021) Immunogenicity, safety, and reactogenicity of heterologous COVID-19 primary vaccination incorporating mRNA, viral-vector, and protein-adjuvant vaccines in the UK (Com-COV2): a single-blind, randomised, phase 2, noninferiority trial Lancet 399 36-49 https://doi.org/10.1016/S0140-6736(21)02718-5 PMID: 34883053 PMCID: 8648333

40. Borobia AM, Carcas AJ, and Pérez-Olmeda M, et al (2021) Immunogenicity and reactogenicity of BNT162b2 booster in ChAdOx1-Sprimed participants (CombiVacS): a multicentre, open-label, randomised, controlled, phase 2 trial Lancet 398(10295) 121-130 https:// doi.org/10.1016/S0140-6736(21)01420-3 PMID: 34181880 PMCID: 8233007

41. Administration USFAD Coronavirus (COVID-19) update: FDAAuthorizes ADDITIONAL vaccine dose for certain immunocompromised individuals (2021) [https://www.fda.gov/news-events/press-announcements/coronavirus-covid-19-update-fda-authorizes-additionalvaccine-dose-certain-immunocompromised]

42. COVID-19 vaccines for moderately to severely immunocompromised people (2021) [https://www.cdc.gov/coronavirus/2019-ncov/ vaccines/recommendations/immuno.html] Date accessed: 3/9/21

43. NCCN recommends third COVID-19 dose for patients with cancer (2021) [https://www.medscape.com/viewarticle/957495] Date accessed: $3 / 9 / 21$

44. Joint Committee on Vaccination and Immunisation (JCVI) advice on third primary dose vaccination (2021) [https://www.gov.uk/government/publications/third-primary-covid-19-vaccine-dose-for-people-who-are-immunosuppressed-jcvi-advice/joint-committee-onvaccination-and-immunisation-jcvi-advice-on-third-primary-dose-vaccination] Date accessed: 8/9/21

45. Kamar N, Abravanel F, and Marion O, et al (2021) Three doses of an mRNA Covid-19 vaccine in solid-organ transplant recipients N Engl J Med 385 661-662 https://doi.org/10.1056/NEJMc2108861 PMID: 34161700 PMCID: 8262620 
46. Shroff RT, Chalasani P, and Wei R, et al (2021) Immune responses to two and three doses of the BNT162b2 mRNA vaccine in adults with solid tumors Nat Med 27(11) 2002-2011 https://doi.org/10.1038/s41591-021-01542-z PMID: 34594036

47. Shapiro LC, Thakkar A, and Campbell ST, et al (2022) Efficacy of booster doses in augmenting waning immune responses to COVID-19 vaccine in patients with cancer Cancer Cell 40(1) 3-5 https://doi.org/10.1016/j.ccell.2021.11.006

48. Heudel, Favier B, and Assaad S, et al (2021) Reduced SARS-CoV-2 infection and death after two doses of COVID-19 vaccines in a series of 1503 cancer patients Ann Oncol 32 1443-1444 https://doi.org/10.1016/j.annonc.2021.07.012 PMID: 34333128 PMCID: 8321960

49. Wölfel R, Corman VM, and Guggemos W, et al (2020) Virological assessment of hospitalized patients with COVID-2019 Nature 581(7809) 465-469 https://doi.org/10.1038/s41586-020-2196-x PMID: 32235945

50. Bullard J, Dust K, and Funk D, et al (2020) Predicting infectious severe acute respiratory syndrome coronavirus 2 from diagnostic samples Clin Infect Dis 71(10) 2663-2666 https://doi.org/10.1093/cid/ciaa638 PMID: 32442256 PMCID: 7314198

51. van Kampen JJA, van de Vijver DA, and Fraaij PL, et al (2021) Duration and key determinants of infectious virus shedding in hospitalized patients with coronavirus disease-2019 (COVID-19) Nat Commun 12(1) 267 https://doi.org/10.1038/s41467-020-20568-4 PMID: 33431879 PMCID: 7801729

52. Liu WD, Chang SY, and Wang JT, et al (2020) Prolonged virus shedding even after seroconversion in a patient with COVID-19 J Infect 81(2) 318-356 PMID: 32283159 PMCID: 7151318

53. Aydillo T, Gonzalez-Reiche AS, and Aslam S, et al (2020) Shedding of viable SARS-CoV-2 after immunosuppressive therapy for cancer $N$ Engl J Med 383(26) 2586-2588 https://doi.org/10.1056/NEJMc2031670 PMID: 33259154 PMCID: 7722690

54. Avanzato VA, Matson MJ, and Seifert SN, et al (2020) Case study: prolonged infectious SARS-CoV-2 shedding from an asymptomatic immunocompromised individual with cancer Cell 183(7) 1901e9-1912e9 https://doi.org/10.1016/j.cell.2020.10.049

55. Centers for Disease Control and Prevention (2021) Interim guidance on duration of isolation and precautions for adults with Covid-19 [https://www.cdc.gov/coronavirus/2019-ncov/hcp/duration-isolation.html] Date accessed: 29/8/21

56. Regev-Yochay G, Amit S, and Bergwerk M, et al (2021) Decreased infectivity following BNT162b2 vaccination: a prospective cohort study in Israel Lancet Reg Health Eur 7100150 https://doi.org/10.1016/j.lanepe.2021.100150 PMID: 34250518 PMCID: 8261633

57. Levine-Tiefenbrun M, Yelin I, and Katz R, et al (2021) Initial report of decreased SARS-CoV-2 viral load after inoculation with the BNT162b2 vaccine Nat Med 27(5) 790-792 https://doi.org/10.1038/s41591-021-01316-7 PMID: 33782619

58. Rouw A, Wexler A, and Kates J, et al (2021) Tracking global covid-19 vaccine equity [https://www.kff.org/coronavirus-covid-19/issuebrief/tracking-global-covid-19-vaccine-equity/] Date accessed: 3/9/21

59. Duan Y, Shi J, and Wang Z, et al (2021) Disparities in COVID-19 vaccination among low-, middle-, and high-income countries: the mediating role of vaccination policy Vaccines (Basel) 9(8) 905 https://doi.org/10.3390/vaccines 9080905

60. Qunaibi EA, Helmy M, and Basheti I, et al (2021) A high rate of COVID-19 vaccine hesitancy in a large-scale survey on Arabs Elife 10 e68038 https://doi.org/10.7554/eLife.68038 PMID: 34042047 PMCID: 8205489

61. Wagner AL, Masters NB, and Domek GJ, et al (2019) Comparisons of vaccine hesitancy across five low- and middle-income countries Vaccines (Basel) 7(4) 155 https://doi.org/10.3390/vaccines7040155

62. Villarreal-Garza C, Vaca-Cartagena BF, and Becerril-Gaitan A, et al (2021) Attitudes and factors associated with COVID-19 vaccine hesitancy among patients with breast cancer JAMA Oncol 7(8) 1242-1244 https://doi.org/10.1001/jamaoncol.2021.1962 PMID: 34110371 PMCID: 8193544

63. Roeker LE, Knorr DA, and Thompson MC, et al (2021) COVID-19 vaccine efficacy in patients with chronic lymphocytic leukemia Leukemia 35(9) 2703-2705 https://doi.org/10.1038/s41375-021-01270-w PMID: 33986431 PMCID: 8118367 
64. Parry H, Mcllroy G, and Bruton R, et al (2021) Antibody responses after first and second Covid-19 vaccination in patients with chronic lymphocytic leukaemia Blood Cancer J 11(7) 136 https://doi.org/10.1038/s41408-021-00528-x PMID: 34330895 PMCID: 8323747

65. Benjamini O, Rokach L, and Itchaki G, et al (2021) Safety and efficacy of BNT162b mRNA Covid19 vaccine in patients with chronic lymphocytic leukemia Haematologica https://doi.org/10.3324/haematol.2021.279196 PMID: 34320789 


\section{Appendix A. Literature search strategy in MEDLINE.}

1 exp *Antibodies, Neutralising/bl, im [Blood, Immunohalogy]

2 COVID-19 Vaccines/ae, bl, im, tu, to [Adverse Effects, Blood, Immunology, Therapeutic Use, Toxicity]

3 exp *Antibodies, Viral/bl, im [Blood, Immunology]

$4 \quad \exp { }^{*}$ COVID-19/bl, im, pc [Blood, Immunology, Prevention \& Control]

5 exp *SARS Virus/im [Immunology]

6 exp *Neoplasms/

$7 \quad 1$ or 2 or 3 or 4 or 5

$8 \quad 6$ and 7

9 exp *Immunogenicity, Vaccine/de, im [Drug Effects, Immunology]

107 or 9

116 and 10 